\title{
Spectral Distribution of the Light Scattered from Rodlike Macromolecules in Solution. II. The Effect of Optical Anisotropy
}

\author{
Hideatsu MAEDA \\ The Institute of Physical and Chemical Research, \\ Wako, Saitama, 351, Japan. \\ Nobuhiko SAITô \\ Department of Applied Physics, Waseda University, \\ Tokyo, 160, Japan.
}

(Received June 3, 1972)

\begin{abstract}
The theory of the spectrum of the light scattered from rodlike macromolecules presented earlier (H. Maeda and N. Saitô, J. Phys. Soc. Japan, 27, 984 (1969).) is extended to optically anisotropic rodlike molecules. The spectrum is shown to be the sum of an infinite number of Lorentzians. The forward scattering is shown to be free from the effect of translational diffusion. The numerical calculations for TMV solution are presented.
\end{abstract}

KEY WORDS Spectral Intensity / Light Scattering / Optical Anisotropy / Rodlike Macromolecule / TMV /

The Brownian motions of macromolecules in solution can be investigated through the spectrum of the scattered light. Thus many theoretical $^{1-5}$ as well as experimental ${ }^{6-10}$ studies on polymer solutions have been carried out. The previous paper ${ }^{4}$ presented a theory for optically isotropic rodlike macromolecules and showed that the coupling between rotatory and translational Brownian motions in rodlike macromolecules is important especially for large scattering angles when the length of the macromolecules is large compared with the wave length of the incident light. This fact was confirmed experimentally by Fujime. $^{10}$ In this paper a further calculation for rodlike macromolecules is provided taking into account optical anisotropy.

\section{COORDINATE SYSTMS}

The amplitude of the electromagnetic wave scattered from a scatterer at the origin is proportional to

$$
\begin{aligned}
\boldsymbol{E}_{\mathrm{S}} & =-\frac{\exp \left[-i \boldsymbol{K} \boldsymbol{R}_{0}\right]}{4 \pi \boldsymbol{R}_{0} \varepsilon}\left[\boldsymbol{K}_{f} \times\left[\boldsymbol{K}_{f} \times \boldsymbol{\mu}\right]\right] \\
\boldsymbol{K} & =\boldsymbol{K}_{\boldsymbol{f}}-\boldsymbol{K}_{0}
\end{aligned}
$$

where $K_{f}, K_{0}$ are the wave vectors of the scat- tered and incident light respectively, $\boldsymbol{\mu}$ is the induced electric dipole moment of the scatterer and $\boldsymbol{R}_{0}$ indicates the position of the observer.

Now if one takes a coordinate system $(x, y, z)$ fixed in space having the origin 0 in the solution. The $z$-axis is taken parallel to the electric vector $\boldsymbol{E}_{0}$ of the incident light, and the angles between $x$-axis and the direction $K_{0}$ and between $z$-axis and $\boldsymbol{K}_{f}$ are denoted as $\Psi_{0}$ and $\Theta_{0}$ respectively. The scattered light is observed in the $x z$-plane in the direction $\boldsymbol{K}_{f}$ with an analyser which makes an angle $\Phi_{0}$ between its polarizing direction and the $x z$-plane, as shown in Figure 1. Then the component $X$ of the electricvector $\boldsymbol{E}_{\mathrm{s}}$ parallel to the direction of the analyser is given by

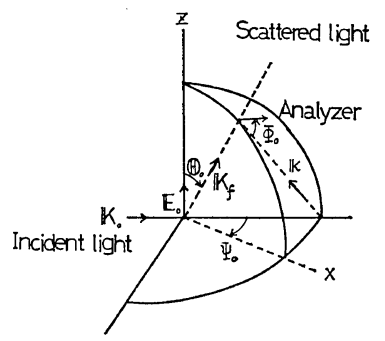

Figure 1. Coordinate systems. 


$$
\begin{aligned}
X= & -\left[{k_{f}}^{2} \exp \left(-i \boldsymbol{K} \boldsymbol{R}_{0}\right) / 4 \pi \boldsymbol{R}_{0} \varepsilon\right] \\
& \otimes\left\{\cos \Phi_{0}\left[\mu_{x} \cos \Theta_{0}-\mu_{z} \sin \Theta_{0}\right]+\mu_{y} \sin \Phi_{0}\right\}
\end{aligned}
$$

where $\mu_{x}, \mu_{y}, \mu_{z}$ are respectively the components of $\mu$ in $x y z$-coordinate system. The polarizability tensor $\boldsymbol{\alpha}$ of the scatterer in the coordinate system $(\xi, \eta, \zeta)$ fixed on the scatterer is written as

$$
\begin{aligned}
\boldsymbol{\alpha} & =\left(\begin{array}{ccc}
\alpha_{\xi \xi} & 0 & 0 \\
0 & \alpha_{\eta \eta} & 0 \\
0 & 0 & \alpha_{\zeta \zeta}
\end{array}\right) \\
& =\left(\alpha_{0}-\alpha_{2} / 3\right) \mathbf{1}+\alpha_{2}\left(\begin{array}{lll}
0 & 0 & 0 \\
0 & 0 & 0 \\
0 & 0 & 1
\end{array}\right)
\end{aligned}
$$

where it is assumed

and put

$$
\alpha_{\xi \xi}=\alpha_{\eta \eta}
$$

$$
\begin{aligned}
& \alpha_{0}=\frac{1}{3}\left(\alpha_{\xi \xi}+\alpha_{\eta \eta}+\alpha_{\zeta \zeta}\right) \\
& \alpha_{2}=\left(\alpha_{\zeta \zeta}-\alpha_{\xi \xi}\right)
\end{aligned}
$$

Let $T$ be the transformation matrix between the $x y z$-system and the coordinate system $(\xi, \eta, \zeta)$ fixed on the scatterer, then one has

$$
\left(\begin{array}{l}
\mu_{y} \\
\mu_{x} \\
\mu_{z}
\end{array}\right)=T \alpha T^{-1}\left(\begin{array}{c}
0 \\
0 \\
E_{0}
\end{array}\right)
$$

Here in order to use the distribution function for the position and orientation of the molecule obtained in the previous paper, one introduces another coordinate system $\left(x^{\prime}, y^{\prime}, z^{\prime}\right)$ with the $z^{\prime}$-axis parallel to $\boldsymbol{K}$ and $x^{\prime}$-axis in the plane containing the direction of $K$ and the $z$-axis with the origin 0 . The orientation of the molecule is described by the polar angle $\theta, \varphi$ (or simply $\Omega$ ) in the coordinate system $\left(x^{\prime}, y^{\prime}, z^{\prime}\right)$. Hence the transformation matrix is rewritten as,

$$
\begin{aligned}
T & =T_{1} T_{2} \\
T_{1} & =\left(\begin{array}{ccc}
\cos \gamma \cos \phi & -\cos \gamma \sin \phi & -\sin \gamma \\
\sin \phi & \cos \phi & 0 \\
\sin \gamma \cos \phi & -\sin \gamma \sin \phi & \cos \gamma
\end{array}\right) \\
T_{2} & =\left(\begin{array}{ccc}
\cos \theta \cos \varphi & \sin \varphi \cos \theta & -\sin \theta \\
-\sin \varphi & \cos \varphi & 0 \\
\sin \theta \cos \varphi & \sin \theta \sin \varphi & \cos \theta
\end{array}\right)
\end{aligned}
$$

where $T_{1}$ and $T_{2}$ are transformation matrices between $(x, y, z)$ and $\left(x^{\prime}, y^{\prime}, z^{\prime}\right)$ and between $\left(x^{\prime}\right.$, $\left.y^{\prime}, z^{\prime}\right)$ and $(\xi, \eta, \zeta)$ systems respectively, and $\gamma$ and $\phi$ are the angles between the direction of $K$ and the $z$-axis, and between the $x$-axis and the $z^{\prime} x^{\prime}$-plane respectively. Substituting eq 2.5 and 2.6 into eq 2.2 , one has

$$
\begin{gathered}
X=K \widetilde{X} \\
K=-k_{f}^{2} \exp \left(-i K \boldsymbol{R}_{0}\right) / 4 \pi \boldsymbol{R}_{0} \varepsilon \\
\widetilde{X}=-\alpha_{2} E_{0}\left\{A \sin \theta \cos \theta \cos \varphi+B \cos ^{2} \theta\right. \\
+C \sin ^{2} \theta \cos ^{2} \varphi+H \sin ^{2} \theta \sin \varphi \cos \varphi \\
+M \sin \theta \cos \theta \sin \varphi\} \\
+\left(\alpha_{0}-\frac{1}{3} \alpha_{2}\right) D E_{0}
\end{gathered}
$$

where the coefficients $A, B, C, H, M$, and $D$ are given in Appendix A. They are functions of $\Theta_{0}, \Psi_{0}$, and $\Phi_{0}$ and the polarizabilities $\alpha_{0}$ and $\alpha_{2}$.

The rodlike molecule considered here is represented by an array of $N$ identical beads (or scatterers) connected in a straight line. The beads are anisotropic scatterers with the anisotropic axis in the direction of the molecule. The position of the $i$ th bead and the center of gravity at time $t$ are designated as $\boldsymbol{R}_{\mathrm{i}}(t)$ and $\boldsymbol{r}(t)$ respectively in the $x^{\prime} y^{\prime} z^{\prime}$-system. Put

$$
\boldsymbol{r}_{\mathrm{i}}(t)=\boldsymbol{R}_{\mathrm{i}}(t)-\boldsymbol{r}(t)
$$

and let the orientation of the molecule be $\Omega$ in polar coordinates as mentioned above. The distribution function $f(r, \Omega, t)$ has already been obtained, ${ }^{4}$ especially the function $f\left(r, \Omega, t \mid \Omega_{0}\right)$ of the molecule at time $t$ under the initial condition that its center of gravity lies at the origin with its orientation $\Omega_{0}\left(\theta_{0}, \varphi_{0}\right)$. In the following, use will be made of the functions $\bar{g}$ and $g$ defined as

$$
\begin{aligned}
\bar{g} & =\mathrm{e}^{k^{2} D_{\mathrm{t}} t} g\left(\boldsymbol{K}, \Omega, t \mid \Omega_{0}\right) \\
& =\mathrm{e}^{k^{2} D_{t} t} \int \mathrm{d} \boldsymbol{r} \mathrm{e}^{i \boldsymbol{r} K} f\left(\boldsymbol{r}, \Omega, t \mid \Omega_{0}\right)
\end{aligned}
$$

where $D_{t}$ is the average translational diffusion constant $k T\left(B_{1}+2 B_{2}\right) / 3$ as given in ref 4 .

\section{SPECTRAL INTENSITY OF SCATTRED LIGHT}

The spectral intensity $I$ of the light scattered 


\section{Optical Anisotropic Light Scattering}

from the rod-like macromolecule as described in ref 2 is given by ${ }^{2}$

$$
\begin{aligned}
I(\Delta \omega, & \left.\Phi_{0}, \Theta_{0}, \Psi_{0}\right) K^{-2} \\
= & \int_{-\infty}^{+\infty}\left\langle\sum_{l, m} \widetilde{X}(t, m) \widetilde{X}^{*}(0, l)\right. \\
& \left.\times \exp \left[i K\left(\boldsymbol{R}_{l}(0)-\boldsymbol{R}_{m}\right)\right]\right\rangle \exp (-i \Delta \omega t) \mathrm{d} t \\
= & \int_{-\infty}^{+\infty} \mathrm{d} t \exp \left[-\left(i \Delta \omega-\boldsymbol{K}^{2} D_{t}\right) t\right] \sum_{l, m} \\
& \times \int \mathrm{d} \Omega \mathrm{d} \Omega_{0} \frac{1}{4 \pi V} \tilde{X}(t, l) \tilde{X}^{*}(0, m) \\
& \times \exp \left[-i K\left(\boldsymbol{r}_{l}(0)-\boldsymbol{r}_{m}\right)\right] \bar{g}\left(t, \boldsymbol{K}, \Omega \mid \Omega_{0}\right) \\
= & \int_{-\infty}^{+\infty} \mathrm{d} t \exp \left[\left(-i \Delta \omega-D_{t} \boldsymbol{K}^{2}\right) t\right] \sum_{l, m} \\
& \times \int_{\mathrm{d}} \mathrm{d} \Omega \mathrm{d} \Omega_{0} \frac{1}{4 \pi V} \bar{g}\left(t, \boldsymbol{K}, \Omega \mid \Omega_{0}\right) \tilde{X}(t, l) \tilde{X}^{*}(0, m) \\
& \times \sum_{v} i^{v}\left(v+\frac{1}{2}\right)\left(\frac{2 \pi}{k r_{l}}\right)^{1 / 2} J_{v+1 / 2}\left(k r_{l}\right) P_{v}\left(\cos \theta_{0}\right) \\
& \times \sum_{u}(-i)^{u}\left(u+\frac{1}{2}\right)\left(\frac{2 \pi}{k r_{m}}\right)^{1 / 2} J_{u+1 / 2}\left(k r_{m}\right) \\
& \times P_{u}(\cos \theta)
\end{aligned}
$$

The function $g$ can be written $a^{4}$

$$
\begin{aligned}
\bar{g}= & \mathscr{L}^{-1} q(\boldsymbol{P})=\frac{1}{2 \pi i} \int_{-\infty}^{+\infty} \mathrm{e}^{P t} q(\boldsymbol{P}) \mathrm{d} \boldsymbol{P} \\
q(\boldsymbol{P})= & \sum \varepsilon^{k}\left(\boldsymbol{a}_{n m}^{(k)}(\boldsymbol{P}) \cos m \varphi\right. \\
& \left.+\boldsymbol{b}_{n m}^{(k)}(\boldsymbol{P}) \sin m \varphi\right) \boldsymbol{P}_{n}{ }^{m}(\cos \theta \\
\varepsilon= & 3\left(\boldsymbol{B}_{3}-\boldsymbol{B}_{1}\right) /\left(2 \boldsymbol{B}_{1}+\boldsymbol{B}_{3}\right)
\end{aligned}
$$

where $B_{1}$ and $B_{3}$ are the mobilities along the $\xi$ and $\zeta$ directions of the molecule, and the coefficients $a_{n m}^{(k)}(p)$ and $b_{n m}^{(l)}(p)$ are given in Appendix B. Substitution eq 3.4 and 3.5 into eq 3.3 gives finally:

$$
\begin{aligned}
I(\Delta \omega & \left., \Phi_{0}, \Theta_{0}, \Psi_{0}\right) K^{-2} \\
= & \pi^{2} E_{0}{ }^{2} \sum_{k}\left(\varepsilon K^{2} D\right)^{k} \sum_{n, \text { even }} L_{f} \mathscr{L}^{-1}\left[\alpha _ { 2 } { } ^ { 2 } \left(A^{2}\right.\right. \\
& \left.+M^{2}\right) X_{n}\left[X_{n}\right]_{k}+\frac{1}{4} \alpha_{2}{ }^{2}\left(C^{2}+H^{2}\right) Y_{n}\left[Y_{n}\right]_{k} \\
& \left.+\left\{\tilde{A}\left[J_{n}\right]-\widetilde{B}\left[K_{n}\right]\right\}\left\{\tilde{A} J_{n}-\widetilde{B} K_{n}\right\}\right]
\end{aligned}
$$

$L_{f}$ is the operator defined by

$$
L_{f} u(t)=\int_{-\infty}^{+\infty} u(|t|) \exp -\left[i \Delta \omega t+k^{2} D_{t}|t|\right] \mathrm{d} t
$$

The functions $X_{n}, Y_{n},\left[X_{n}\right]_{k},\left[Y_{n}\right]_{k}, J_{n}, k_{n}$ and $\left[J_{n}\right],\left[K_{n}\right]$ are listed in Appendix C. Further the following definitions are introduced

$$
L_{f} \mathscr{L}^{-1}\left[W_{n}\right]_{l}=\left[\left[W_{n}\right]\right]_{l}
$$

where $W_{n}=X_{n}, Y_{n}, J_{n}$, and $K_{n}$. Using these quantities one can rewrite eq 3.6 as

$$
\begin{aligned}
I(\Delta \omega, & \left.\Phi_{0}, \Theta_{0}, \Psi_{0}\right) K^{-2} \\
= & \pi E_{0}{ }^{2} \sum_{l}\left(\varepsilon \boldsymbol{K}^{2} D_{t}\right)^{l} \sum_{n, \text { even }}\left[\alpha_{2}{ }^{2}\left(A^{2}+M^{2}\right) X_{n}\left[\left[X_{n}\right]\right]_{l}\right. \\
& +\frac{1}{4} \alpha_{2}{ }^{2}\left(C^{2}+H^{2}\right) Y_{n}\left[\left[Y_{n}\right]\right]_{l} \\
& \left.+\left\{\widetilde{A}\left[\left[J_{n}\right]\right]_{l}-\widetilde{B}\left[\left[K_{n}\right]\right]_{l}\right\}\left\{\tilde{A} J_{n}-\widetilde{B} K_{n}\right\}\right]
\end{aligned}
$$

Explicit expressions of $\left[\left[W_{n}\right]\right]_{l}$ are given in Appendix C. Equation 3.9 gives the spectral intensity of the scattered light in any arbitrary direction $\left(\Theta_{0}, \Psi_{0}, \Phi_{0}\right)$.

\section{RESULTS AND DISCUSSION}

(i) The spectral intensity of the scattered light consists of terms proportional to $\alpha_{0}{ }^{2}$ and $\alpha_{2}{ }^{2}$ as well as $\alpha_{0} \alpha_{2}$. The last term does not exist in the anisotropic flexible chain, and further in rodlike molecules of the length less than the wave length of the incident light as treated by Pecora. This fact was already pointed out by Tagami. ${ }^{11}$

(ii) Pecora $^{2}$ showed that the isotropic part is a single Lorentzian and the anisotrpic part consists of five Lorentzian terms in the case of rods whose length is short compared with the wave length of the light. In larger rodlike molecules, however, both the isotropic and the anisotropic parts consist of infinite numbers of Lorentzians.

(iii) The coupling between translational and rotational Brownian motions gives rise to an effect similar to the spectral intensity for both the optically anisotropic and isotropic rodlike molecules.

For numerical calculations one uses the values of tabacco mesaic virus, i.e., $3000 \AA$ in length, $150 \AA$ in diameter, $390 \mathrm{sec}^{-1}$ in the rotational diffusion constant and $0.3 \times 10^{-7} \mathrm{~cm}^{2} / \mathrm{sec}$ in the translational diffusion constant. The results are given in Figures 2-6, where the ordinate scales are arbitrary. In Figure 2, one takes $\Theta_{0}=160.4^{\circ}$, $\Phi_{0}=0$ and $\alpha_{2} / \alpha_{0}=0$ and 0.4 , and $D_{t}=1 \times 10^{-7}$ $\mathrm{cm}^{2} / \mathrm{sec}$. The full and dotted lines show the spectra with and without the coupling between the translational and the rotational Brownian motions respectively. It is clear that, in the case of Figure 2, the effect of the coupling is by no 


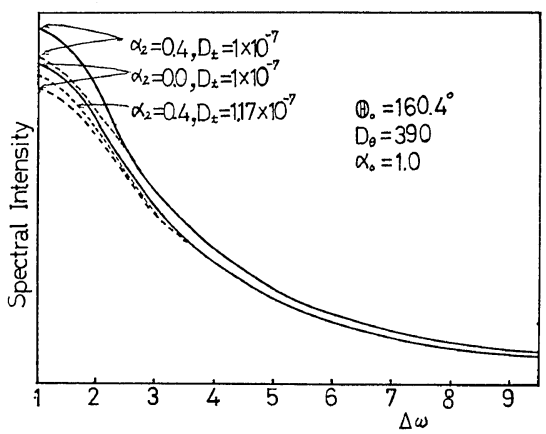

Figure 2. Spectra of the scattered lights from anisotropic rodlike macromolecules compared with those from isotropic ones. Full lines: Results taking into account the coupling of the rotatory and translational Browniam motions, Broken lines: Results without taking the coupling into accout.

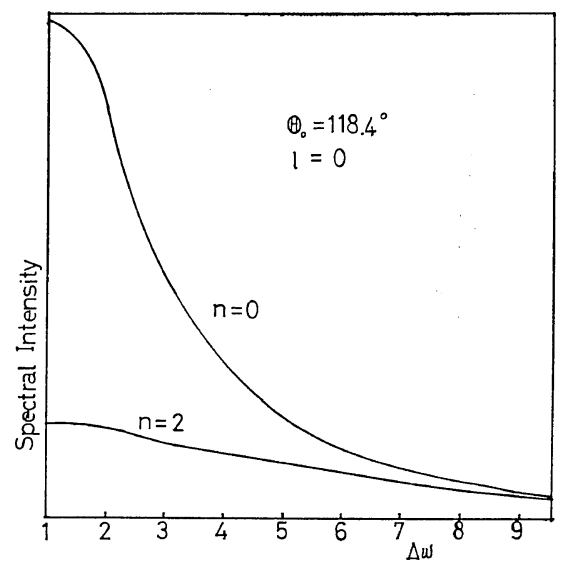

Figure 3. Contribution of the relaxation modes $l=0$ and $n=0,2$ at $\Theta_{0}=118.4^{\circ}$ to the total spectral intensity.

means negligible and also the optical anisotropy gives aise to an increase in the spectral intensity. One sees further that the higher the value of the diffusion constant $D_{t}$, the smaller the intensity.

In Figure 3, one takes scattering angle $\Theta_{0}=$ $118.4^{\circ}$ and $l=0$. The term $l=0$ corresponds to the case free from coupling between the translational and rotatory diffusions. The number refers to the relaxation mode number, which is related to the relaxation time as $\left[D_{t} k^{2}+D_{\theta} n(n+\right.$ $1)]^{-1}$. The term for $n \geqq 4$ is negligibly small. Figure 4 is the similar one for $\Theta_{0}=160.4^{\circ}$ and $l=0$. One sees that the larger the scattering

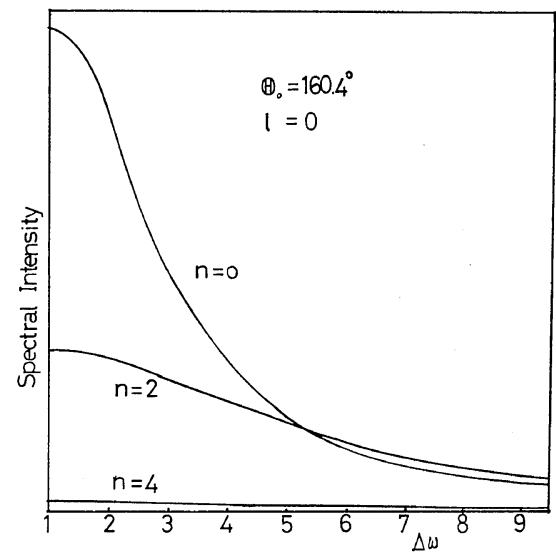

Figure 4. Contribution of the relaxation modes $l=0$ and $n=0,2,4$ at $\Theta_{0}=160.4^{\circ}$ to the total spectral intensity.

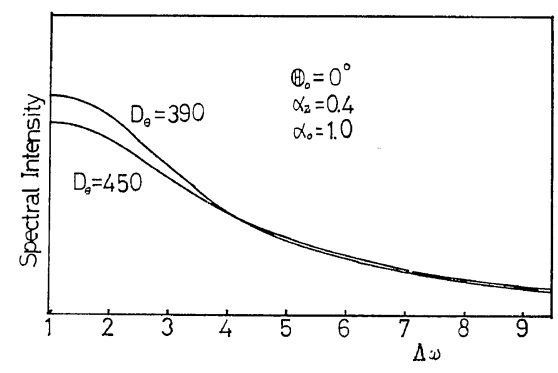

Figure 5. Spectral intesity of the molecules with different rotational diffusion constants in the forward scattering.

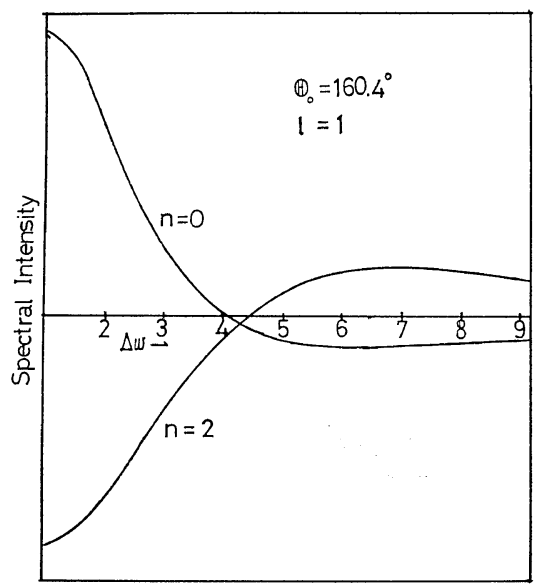

Figure 6. Contribution of the relaxation modes $l=1$ and $n=0,2$ to the total spectral intensity. The curve for $n=2$ is drawn ten times larger in ordinate than the one for $n=0$. 


\section{Optical Anisotropic Light Scattering}

angle the larger the contribution of the rotational relaxation to the total intensity. In Figure 5 one takes $\Theta_{0}=0^{\circ}, \Phi_{0}=90^{\circ}$ (forward scattering with crossed polarizer and analyser), $\alpha_{2} / \alpha_{0}=0.40$ and $D_{\theta}=390 / \mathrm{sec}$ or $450 / \mathrm{sec}$. In this case the rotational Brownian motion only is observed in the spectrum. ${ }^{9}$ In general the spectral intensity is composed of terms of various sets of $n$ and $l$. In Figure 6, terms for $l=1$ and $n=0$, 2 , are given. Figure 2 is the sum of all $(n, l)$ terms.

\section{APPENDIX A}

$$
\begin{array}{rl}
\tilde{A}=2 & B-C \\
= & \frac{1}{2\left(1-\sin \Theta_{0}\right) \cos \Psi_{0}}\left\{\left(3-\sin ^{2} \Theta_{0}\right) \cos \Psi_{0} \cos \Phi_{0}\right. \\
& \left.+3 \cos \Theta_{0} \sin \Phi_{0} \sin \Psi_{0}-2 \sin \Theta_{0} \cos \Phi_{0}\right\} \\
\widetilde{B}= & -2 D\left(\alpha_{0}+\alpha_{2} / 3\right)-C \alpha_{2} \\
= & \left\{2\left(\alpha_{0}+\alpha_{2} / 3\right) \sin \Theta_{0} \cos \Phi_{0}\right\} \\
& -\frac{\alpha_{2}}{2\left(1-\sin \Phi_{0} \sin \Psi_{0}\right)^{2}}\left\{2 \sin \Theta_{0} \cos \Phi_{0}\right. \\
& -\left(1+\sin \Theta_{0}\right) \cos \Phi_{0} \cos \Psi_{0} \\
& \left.-\cos \Theta_{0} \sin \Psi_{0} \sin \Phi_{0}\right\} \\
M^{2}+ & A^{2} \\
= & \frac{\left(\sin \Phi_{0} \cos \Psi_{0}-\sin \Phi_{0} \sin \Theta_{0}-\sin _{0} \cos \Phi_{0} \cos \Theta_{0}\right)^{2}}{2\left(1-\sin \Theta_{0} \cos \Psi_{0}\right)^{2}\left(1+\sin ^{2} \Phi_{0}-2 \sin \Theta_{0} \cos \Psi_{0}\right)} \\
\times\{ & \sin \Theta_{0} \cos \Psi_{0}+\sin \Theta_{0}\left(2 \sin \Psi_{0}-1\right) \\
- & \left.\sin \Theta_{0} \cos \Psi_{0}+1\right\} \\
C^{2}= & \frac{1}{4\left(1-\sin \Theta_{0} \cos \Psi_{0}\right)^{2}}\left\{2 \sin \Theta_{0} \cos \Phi_{0}\right. \\
& \quad-\left(1+\sin \Theta_{0}\right) \cos \Phi_{0} \cos \Psi_{0} \\
& \left.\quad-\cos \Theta_{0} \sin \Psi_{0} \sin \Phi_{0}\right\}^{2} \\
H^{2}= & \frac{1}{2\left(1-\sin \Theta_{0} \cos \Psi_{0}\right)}\left\{\sin \Psi_{0} \cos \Phi_{0} \cos \Theta_{0}\right. \\
& \left.\quad+\sin \Theta_{0} \sin \Phi_{0}-\sin _{0} \cos \Psi_{0}\right\}^{2}
\end{array}
$$

\section{APPENDIX B}

The explicit expressions of the coefficients $a_{n m}^{l}(p)$ and $b_{n m}^{l}(p)$ are given in ref 4 , and are written in the following way,

$$
a_{n m}^{l} \equiv \sum_{j=-l}^{l} A_{n m}^{l, j} P_{n+2 j}\left(\cos \theta_{0}\right) \cos m \varphi_{0}
$$

and $b_{n m^{\prime}}$ s are given by replacing $\cos m \varphi_{0}$ by $\sin$ $m \varphi_{0}$ with same coefficients. Also the coefficients $A_{n m}^{l, j}$ are defined as follows. For $l=0$ and $l=1$, in particular,

$$
\begin{aligned}
& A_{m, 0}^{0,0}=\varepsilon_{n} \frac{2 n+1}{4 \pi} \\
& A_{n, 1}^{0,0}=\varepsilon_{n} \frac{2 n+1}{2 \pi} \cdot \frac{(n-1) !}{(n+1) !} \\
& A_{n, 2}^{0,0}=\varepsilon_{n} \frac{2 n+1}{2 \pi} \frac{(n-2) !}{(n+2) !} \\
& A_{n+2,0}^{1,1}=-\varepsilon_{n} \varepsilon_{n+2} \frac{(n+1)(n+1)}{4 \pi(2 n+3)} \\
& A_{n, 0}^{1,0}=-\varepsilon_{n}{ }^{2} \frac{1}{4 \pi}\left\{\frac{n^{2}}{(2 n-1)}+\frac{(n+1)^{2}}{(2 n+3)}-\frac{1}{3}(2 n+1)\right\} \\
& A_{n+2,1}^{1,1}=-\varepsilon_{n} \varepsilon_{n+2} \frac{1}{2 \pi(2 n+3)} \\
& A_{n, 1}^{1,0}=-\varepsilon_{n}{ }^{2} \frac{(n-1) !}{2 \pi(n+1) !}\left\{\frac{\left(n^{2}-1\right)}{(2 n-1)}+\frac{n(n+2)}{2 n+3}\right. \\
& \left.\quad-\frac{1}{3}(2 n+1)\right\} \\
& A_{n+2,2}^{1,1}=-\varepsilon_{n} \varepsilon_{n+2} \frac{1}{2 \pi(n+1)(n+2)(n+3)} \\
& A_{n, 2}^{1,0}=-\varepsilon_{n}{ }^{2} \frac{(n-2) !}{2 \pi(n+2) !}\left\{\frac{n^{2}-4}{2 n-1}+\frac{(n-1)(n+3)}{2 n+3}\right. \\
& \left.-\frac{1}{3}(2 n+1)\right\}
\end{aligned}
$$

where

$$
\varepsilon_{n}=\frac{1}{P+D_{\theta} n(n+1)}
$$

\section{APPENDIX C}

$$
\begin{aligned}
J_{n}= & \sum_{s, j} i^{s}\left(s+\frac{1}{2}\right)\left(2 \pi / k r_{j}\right)^{1 / 2} J_{s+1 / 2}\left(k r_{j}\right) \\
& \times \int \cos ^{2} \theta P_{s}(\cos \theta) P_{n}(\cos \theta) \sin \theta \mathrm{d} \theta \\
= & \sum_{j} i^{n}\left(2 \pi / k r_{j}\right)^{1 / 2}\left\{-\frac{(n+1)(n+2)}{(2 n+1)(2 n+3)} J_{n+5 / 2}\left(k r_{j}\right)\right. \\
& +\frac{1}{2 n+1}\left(\frac{n^{2}}{2 n-1}+\frac{(n+1)^{2}}{2 n+3}\right) \\
& \left.\times J_{n+1 / 2}\left(k r_{j}\right)-\frac{n(n-1)}{(2 n-1)(2 n+1)} J_{n-3 / 2}\left(k r_{j}\right)\right\} \\
K_{n}= & \sum_{s, j} i^{s}\left(s+\frac{1}{2}\right)\left(2 \pi / k r_{j}\right)^{1 / 2} J_{s+1 / 2}\left(k r_{j}\right) \\
& \times \int P_{s}(\cos \theta) P_{n}(\cos \theta) \sin \theta \mathrm{d} \theta
\end{aligned}
$$




\section{H. Maeda and N. Saitô}

$$
\begin{aligned}
& =\sum_{j} i^{n}\left(2 \pi / k r_{j}\right)^{1 / 2} J_{s+1 / 2}\left(k r_{j}\right) \\
& X_{n}=\sum_{s, j} i^{s}\left(s+\frac{1}{2}\right)\left(2 \pi / k r_{j}\right)^{1 / 2} J_{s+1 / 2}\left(k r_{j}\right) \\
& \times \int \sin \theta \cos \theta \boldsymbol{P}_{\boldsymbol{s}}(\cos \theta) \boldsymbol{P}_{n}{ }^{1}(\cos \theta) \sin \theta \mathrm{d} \theta \\
& =\sum_{j} i^{n}\left(2 \pi / k r_{j}\right)^{1 / 2} \frac{1}{2 n+1} \cdot \frac{(n+1) !}{(n-1) !} \\
& \times\left\{\frac{2 n+3}{n+2} J_{n+5 / 2}\left(k r_{j}\right)\right. \\
& +\frac{2 n+1}{(2 n-1)(2 n+3)} J_{n+1 / 2}\left(k r_{j}\right) \\
& \left.-\frac{n-1}{2 n-1} J_{n-3 / 2}\left(k r_{j}\right)\right\} \\
& Y_{n}=\sum_{s, j} i^{s}\left(s+\frac{1}{2}\right)\left(2 \pi / k r_{j}\right)^{1 / 2} J_{s+1 / 2}\left(k r_{j}\right) \\
& \times \int \sin ^{2} \theta \boldsymbol{P}_{s}(\cos \theta) \boldsymbol{P}_{n}{ }^{2}(\cos \theta) \sin \theta \mathrm{d} \theta \\
& =\sum_{j} i^{n}\left(2 \pi / k r_{j}\right)^{1 / 2} \frac{1}{2 n+1} \frac{(n+2) !}{(n-2) !} \\
& \times\left\{-\frac{1}{2 n+3} J_{n+5 / 2}\left(k r_{j}\right)\right. \\
& -\frac{2(2 n+1)}{(2 n-1)(2 n+3)} J_{n+1 / 2}\left(k r_{j}\right) \\
& \left.-\frac{1}{2 n-1} J_{n-3 / 2}\left(k r_{j}\right)\right\} \\
& {\left[W_{n}\right]_{l}=\sum_{j=0}^{l}\left(2-\delta_{j, 0}\right) A_{n+2 j, r}^{l, j} W_{n+2 j}}
\end{aligned}
$$

where $\delta_{j, 0}$ is Kronecker's delta function and the suffix $r$ in $A_{n+2 j, r}^{l, j}$ is defined as,

$$
\begin{gathered}
r=\left\{\begin{array}{lll}
0 & \text { for } & W_{n}=J_{n}=K_{n} \\
1 & \text { for } & W_{n}=X_{n} \\
2 & \text { for } & W_{n}=Y_{n}
\end{array}\right. \\
{\left[\left[W_{n}\right]\right]_{l} \equiv \sum_{j=0}^{l} u_{j}^{l}(w) W_{n+2 j}}
\end{gathered}
$$

Inparticular for $l=0$,

$$
\begin{aligned}
{\left[\left[J_{n}\right]\right]_{0}=} & \frac{2 n+1}{4 \pi} \cdot \frac{\left[D_{\theta} n(n+1)+D_{t} k^{2}\right]}{\left[D_{\theta} n(n+1)+D_{t} k^{2}\right]^{2}+\Delta \omega^{2}} J_{n} \\
{\left[\left[K_{n}\right]\right]_{0}=} & \frac{2 n+1}{4 \pi} \cdot \frac{2\left[D_{\theta} n(n+1)+D_{t} k^{2}\right]}{\left[D_{\theta} n(n+1)+D_{t} k^{2}\right]^{2}+\Delta \omega^{2}} K_{n} \\
{\left[\left[X_{n}\right]\right]_{0}=} & \frac{2 n+1}{2 \pi} \cdot \frac{(n-1) !}{(n+1) !} \\
& \times \frac{2\left[D_{\theta} n(n+1)+D_{t} k^{2}\right]}{\left[D_{\theta} n(n+1)+D_{t} k^{2}\right]^{2}+\Delta \omega^{2}} X_{n}
\end{aligned}
$$

$$
\begin{aligned}
{\left[\left[Y_{n}\right]\right]_{0}=} & \frac{2 n+1}{2 \pi} \cdot \frac{(n-2) !}{(n+2) !} \\
& \times \frac{2\left[D_{\theta} n(n+1)+D_{t} k^{2}\right]}{\left[D_{\theta} n(n+1)+D_{t} k^{2}\right]^{2}+\Delta \omega^{2}} Y_{n}
\end{aligned}
$$

For $l=1$, the coefficients $U_{j}^{1}(w)$ are given as follows,

$$
\begin{aligned}
& U_{1}^{1}(J)=-\frac{2(n+1)(n+2)}{4 \pi(2 n+3)} \omega_{3} \\
& U_{0}^{1}(J)=-\frac{1}{4 \pi}\left\{\frac{n^{2}}{2 n-1}+\frac{(n+1)^{2}}{2 n+3}\right. \\
& \left.-\frac{1}{3}(2 n+1)\right\} \omega_{2} \\
& U_{1}^{1}(X)=-\frac{2}{2 \pi(2 n+3)} \omega_{2} \\
& U_{0}^{1}(X)=-\frac{1}{2 \pi} \frac{(n-1) !}{(n+1) !}\left\{\frac{(n-1)(n+1)}{2 n-1}\right. \\
& \left.+\frac{n(n+2)}{2 n+3}-\frac{1}{3}(2 n+1)\right\} \omega_{2} \\
& U_{1}^{1}(Y)=-\frac{2}{2 \pi(n+1)(n+2)(n+3)} \omega_{3} \\
& U_{0}^{1}(Y)=-\frac{1}{2 \pi} \cdot \frac{(n-2) !}{(n+2) !}\left\{\frac{(n-2)(n+2)}{(2 n-1)}\right. \\
& \left.+\frac{(n-1)(n+3)}{(2 n+3)}-\frac{1}{3}(2 n+1)\right\} \omega_{3}
\end{aligned}
$$

where

$$
\begin{aligned}
\omega_{1}= & \frac{2\left[D_{\theta} n(n+1)+D_{t} k^{2}\right]}{\left[D_{\theta} n(n+1)+D_{t} k^{2}\right]^{2}+\Delta \omega^{2}} \\
\omega_{2}= & \frac{2\left\{\left[D_{\theta} n(n+1)+D_{t} k^{2}\right]^{2}-\Delta \omega^{2}\right\}}{\left\{\left[D_{\theta} n(n+1)+D_{t} k^{2}\right]^{2}+\Delta \omega^{2}\right\}^{2}} \\
\omega_{3}= & \frac{1}{(2 n+3) D_{\theta}}\left\{\frac{D_{\theta} n(n+1)+D_{t} k^{2}}{\left[D_{\theta} n(n+1)+D_{t} k^{2}\right]^{2}+\Delta \omega^{2}}\right. \\
& \left.-\frac{D_{\theta}(n+2)(n+3)+D_{t} k^{2}}{\left[D_{\theta}(n+2)(n+3)+D_{t} k^{2}\right]^{2}+\Delta \omega^{2}}\right\}
\end{aligned}
$$

\section{REFERENCES}

1. R. Pecora, J. Chem. Phys., 40, 1604 (1964); ibid., 48, 4126 (1968); ibid., 49, 1032 (1968).

2. R. Pecora, J. Chem. Phys., 49, 1036 (1968).

3. N. Saitô and S. Itô, J. Phys. Soc. Japan, 25, 1446 (1968).

4. H. Maeda and N. Saitô, J. Phys. Soc. Japan, 27, 984 (1969).

5. Y. Tagami and R. Pecora, J. Chem. Phys., 51, 3293 and 3298 (1969). 


\section{Optical Anisotropic Light Scattering}

6. H. Z. Cummins, N. Knable, and Y. Yeh, Phys. Rev. Letters, 12, 150 (1964).

7. S. B. Dubin, J. H. Lunacek, and G. B. Benedek, Proc. Natl. Acad. Sci. U. S., 57, 1164 (1967).

8. H. Z. Cummins, F. D. Carlson, T. J. Herbert, and G. Woods, Biophys. J., 9, 518 (1969).
9. A. Wada, N. Suda, T. Tsuda, and K. Soda, J. Chem. Phys., 50, 31 (1969).

10. S. Fujime, J. Phys. Soc. Japan, 29, 416, 751 (1970).

11. Y. Tagami, J. Chem. Phys., 54, 4990 (1971). 